\title{
Fuzzy Robust Tracking Control for Uncertain Nonlinear Time-Delay System
}

\author{
Z.-B. Du, T.-C. Lin, T.-B. Zhao
}

\author{
Zhenbin Du \\ Yantai University \\ Yantai, Shandong, 264005, China \\ zhenbindu@126.com \\ Tsung-Chih Lin \\ Feng-Chia University \\ Taichung, 40724, Taiwan \\ tclin@fcu.edu.tw \\ Tiebiao Zhao \\ University of California \\ Merced, CA, 95343, USA \\ tzhao3@ucmerced.edu
}

\begin{abstract}
The problem of fuzzy robust tracking control is investigated for uncertain nonlinear time-delay systems. The nonlinear time-delay system is modeled as fuzzy Takagi-Sugeno (T-S) system, and fuzzy logic systems are used to eliminate the uncertainties of the system. A sufficient condition for the existence of fuzzy controller is given in terms of linear matrix inequalities (LMIs) and adaptive law. Based on Lyapunov stability theorem, the fuzzy control scheme guarantees the desired tracking performance in sense that all the closed-loop signals are uniformly ultimately bounded (UUB). Simulation results of 2-link manipulator demonstrate the effectiveness of the developed control scheme.
\end{abstract}

Keywords: fuzzy T-S model; fuzzy logic systems; nonlinear system; time-delay; tracking control.

\section{Introduction}

Fuzzy control approach offers a powerful and systematical control methodology to handle nonlinear system. Owing to the superior approximation and reasoning abilities of the fuzzy controller, fuzzy control approach has been applied in different applications. With the extensive efforts of the researchers working on the fuzzy control discipline, fruitful stability analysis results have been obtained to aid the design of stable fuzzy controllers. In [1], a fuzzy T-S model was employed to represent the system dynamics of the nonlinear system. The fuzzy T-S model represents the nonlinear system as a weighted sum of some linear subsystems. This particular structure offers a general framework to represent the nonlinear system which is favorable for system analysis. Fuzzy controllers [2-4] were proposed to handle the nonlinear system represented by the fuzzy T-S model. To avoid the effect of the uncertainties, a matching condition is assumed in [5-7], and an upper bound on uncertainties is introduced in [8-10]. The matching condition and the upper bound in dealing with the uncertainties are effective and feasible. However, there exists certain conservatism. The matching condition is a very conservative assumption and the upper bound may be too big or too small, which adds some difficulties to the controller design. On the other hand, it is well known that fuzzy logic systems can uniformly approximate nonlinear continuous functions to arbitrary accuracy. Thus, fuzzy logic systems are used to model uncertain nonlinear systems in [11-13]. 
Time delays are frequently encountered in engineering systems. The existence of time delays usually becomes the source of instability and degrading performance of systems. Therefore, stability analysis and controller synthesis for nonlinear time-delay systems are important both in theory and in practice.

By using fuzzy T-S model and fuzzy logic systems, we propose a novel robust tracking control scheme for a class of uncertain nonlinear time-delay system. Fuzzy T-S model is used to approximate the nonlinear system, and a fuzzy state feedback controller is designed to guarantee the stability of the fuzzy system. A compensator based fuzzy logic systems is introduced to eliminate the uncertainties of the system. The fuzzy control scheme ensures the desired tracking performance in sense that all the closed-loop signals are uniformly ultimately bounded (UUB).

The rest of the paper is organized as follows. Section 2 provides the problem formulation. Section 3 develops a procedure of the controller design. Section 4 gives the main result. Section 5 presents simulation examples to illustrate the effectiveness of the proposed method. These are followed by conclusions in Section 6 .

\section{Problem formulation}

Consider the following uncertain nonlinear time-delay system:

$$
\begin{aligned}
& \dot{x}_{1}=x_{2}, \\
& \ldots \\
& \dot{x}_{\left(\beta_{1}-1\right)}=x_{\beta_{1}}, \\
& \dot{x}_{\beta_{1}}=f_{1}\left(x, x\left(t-\tau_{1}\right), \cdots, x\left(t-\tau_{r}\right), u\right)+\tilde{f}_{1}\left(x, x\left(t-\tau_{1}\right), \cdots, x\left(t-\tau_{r}\right), u\right)+d_{1}, \\
& \dot{x}_{\left(\beta_{1}+1\right)}=x_{\left(\beta_{1}+2\right)}, \\
& \quad \cdots \\
& \dot{x}_{n}=f_{m}\left(x, x\left(t-\tau_{1}\right), \cdots, x\left(t-\tau_{r}\right), u\right)+\tilde{f}_{m}\left(x, x\left(t-\tau_{1}\right), \cdots, x\left(t-\tau_{r}\right), u\right)+d_{m},
\end{aligned}
$$

where $x=\left[x_{1}, \cdots, x_{1}^{\left(\beta_{1}-1\right)}, \cdots, x_{\left(n-\beta_{m}+1\right)}, \cdots, x_{\left(n-\beta_{m}+1\right)}^{\left(\beta_{m}-1\right)}\right]^{T} \in R^{n}$ with $\beta_{1}+\beta_{2}+\cdots+\beta_{m}=n$ and $u \in R^{m}$ are the system state and control input, respectively. $f_{i}(i=1, \cdots, m)$ are known smooth nonlinear functions, $\tilde{f}_{i}(i=1, \cdots, m)$ are unknown nonlinear uncertainties, $\tau_{i}(i=1, \cdots, r)$ are time delays, and $d_{i}(i=1, \cdots, m)$ are external bounded disturbances.

The control objective of this paper is to find a fuzzy tracking controller such that, while maintaining all the closed-loop signals UUB, the system states of nonlinear system (1) follow those of the given stable reference model.

\section{Fuzzy model, reference model and fuzzy controller}

A fuzzy-model-based control system, formed by a fuzzy model, a reference model, and fuzzy controller connected in a closed-loop, is introduced.

\subsection{Fuzzy model}

A fuzzy dynamic model has been proposed by Takagi and Sugeno to represent a nonlinear system. The fuzzy dynamic model is described by the following fuzzy IF-THEN rules and will be employed here to deal with the control design problem for the nonlinear system in (1). 
Plant Rule $i$ : IF $z_{1}(t)$ is $F_{1}^{i}$ and, $\cdots$, and $z_{s}(t)$ is $F_{s}^{i}, T H E N$

$$
\dot{x}(t)=A_{i} x(t)+\sum_{l=1}^{r} A_{i l} x\left(t-\tau_{l}\right)+B_{i} u(t)+d, \quad i=1, \cdots, L
$$

where $z_{1}(t), \cdots, z_{s}(t)$ are the premise variables, $F_{j}^{i}(j=1, \cdots, s)$ are the fuzzy sets, $L$ is the number of IF-THEN rules, $A_{i}, B_{i}$ and $A_{i l}$ are some constant matrices with compatible dimensions, $B_{i}=\left[0, \cdots, b_{i 1}^{T}, \cdots, 0, \cdots, b_{i m}^{T}\right]^{T} \in R^{n \times m}$ with $b_{i 1} \in R^{m}, \cdots, b_{i m} \in R^{m}$, and $d=\left[0, \cdots, d_{1}, \cdots, 0, \cdots, d_{m}\right]^{T}$.

Then, the final output of the fuzzy system is inferred as follows:

$$
\dot{x}(t)=\sum_{i=1}^{L} \mu_{i}\left[A_{i} x(t)+\sum_{l=1}^{r} A_{i l} x\left(t-\tau_{l}\right)\right]+\sum_{i=1}^{L} \mu_{i} B_{i} u(t)+d,
$$

where

$$
\mu_{i}=v_{i}(z(t)) / \sum_{i=1}^{L} v_{i}(z(t)), v_{i}(z(t))=\prod_{j=1}^{s} F_{j}^{i}\left(z_{j}(t)\right)
$$

for all $t \geq 0$, and $F_{j}^{i}\left(z_{j}(t)\right)$ is the grade of membership of $z_{j}(t)$ in $F_{j}^{i}$. It can be seen that $\sum_{i=1}^{L} v_{i}(z(t))>0$, and $v_{i} \geq 0(i=1, \cdots, r)$ for all $t \geq 0$. We have $\mu_{i} \geq 0(i=1, \cdots, r), \sum_{i=1}^{L} \mu_{i}=1$.

Hence, the nonlinear system (1) can be rearranged as the following equivalent system :

$$
\dot{x}(t)=\sum_{i=1}^{L} \mu_{i}\left[A_{i} x(t)+\sum_{l=1}^{r} A_{i l} x\left(t-\tau_{l}\right)\right]+\sum_{i=1}^{L} \mu_{i} B_{i} u(t)+B \Delta(x, x(t-\tau))+d,
$$

where $B \Delta(x, x(t-\tau))=B \Delta\left(x, x\left(t-\tau_{1}\right), \cdots, x\left(t-\tau_{r}\right)\right)$ denotes the uncertainties between the nonlinear system (1) and the fuzzy model $(3)$, and $B=\operatorname{diag}\left[B^{1}, \cdots, B^{m}\right]$ with $B^{i}=[0, \cdots, 0,1]^{T} \in$ $R^{\beta_{i}}$.

\subsection{Reference model}

The system states of nonlinear systems (1) are driven to follow those of the following stable reference model

$$
\dot{x}_{r}(t)=A_{r} x_{r}(t)+r(t),
$$

where $x_{r}(t)$ is a reference state, $r(t)$ is a bounded reference input, and $A_{r}$ is an asymptotically stable matrix.

\subsection{Fuzzy controller}

A fuzzy controller is chosen as

$$
u(t)=u_{l}(t)-u_{f}(t)
$$

where $u_{l}(t)$ denotes the fuzzy state feedback control based on T-S model, and $u_{f}(t)$ is the adaptive compensator based on fuzzy logic systems. The former is used to stabilize the linear part of system (11), and the latter is used to compensate the uncertainties. $u_{l}(t)$ and $u_{f}(t)$ are designed as (8) and (10), respectively.

For the fuzzy model represented by (2) or (3), fuzzy state feedback control $u_{l}(t)$ shares the same IF parts with the following structure. 
Control Rule i: IF $z_{1}(t)$ is $F_{1}^{i}$ and, $\cdots$, and $z_{s}(t)$ is $F_{s}^{i}$, THEN

$$
u_{l}(t)=K_{i}\left(x(t)-x_{r}(t)\right), \quad i=1, \cdots, L .
$$

Hence, the overall state feedback controller $u_{l}(t)$ is given by

$$
u_{l}(t)=\sum_{i=1}^{L} \mu_{i} K_{i}\left(x(t)-x_{r}(t)\right)
$$

where $K_{i}(i=1,2, \cdots, L)$ are matrices with proper dimensions and satisfy the following inequalities

$$
\bar{A}_{i j}^{T} P+P \bar{A}_{i j}+\sum_{l=1}^{r} \alpha_{l}^{-1} P \bar{A}_{i l} \bar{A}_{i l}^{T} P+\sum_{l=1}^{r} \alpha_{l} I+\frac{1}{\rho^{2}} P P+\bar{Q}<0, \quad i, j=1, \cdots, L,
$$

where $\bar{A}_{i j}=\left[\begin{array}{ll}A_{i}+B_{i} K_{j} & -B_{i} K_{j} \\ 0 & A_{r}\end{array}\right], \bar{A}_{i l}=\left[\begin{array}{ll}A_{i l} & 0 \\ 0 & 0\end{array}\right], \bar{Q}=\operatorname{diag}\{2 Q, 2 Q\}, P$ and $Q$ are some symmetric and positive definite matrices, and $\alpha_{l}(l=1, \cdots, r)$ are positive constants.

The adaptive compensator based on fuzzy logic systems $u_{f}(t)$ are as follows:

$$
u_{f}(t)=\left\{\begin{array}{l}
E^{-1} \hat{u}(x, x(t-\tau) \mid \Theta), \text { if } E \text { is nonsigular } \\
E^{T}\left(I+E E^{T}\right)^{-1} \hat{u}(x, x(t-\tau) \mid \Theta), \text { if } E \text { is sigular }
\end{array}\right.
$$

where $E_{i}=\left[b_{i 1}^{T}, \cdots, b_{i m}^{T}\right]^{T} \in R^{m \times m}, E=\sum_{i=1}^{L} \mu_{i} E_{i}$, and $\hat{u}(x, x(t-\tau) \mid \Theta)$ is constructed by fuzzy logic systems. The weight $\Theta$ is an adaptive parameter, which is adapted by

$$
\dot{\Theta}=\eta_{1} \Psi^{T}(x, x(t-\tau)) \bar{B}^{T} P \tilde{x},
$$

where $\eta_{1}$ is a positive constant, $\Psi(x, x(t-\tau))$ is a fuzzy basis-function matrix, and $\tilde{x}=\left[x^{T}, x_{r}^{T}\right]^{T}$.

In the following, we explain the solution of the inequalities (9) and the construction of fuzzy logic systems $\hat{u}(x, u \mid \Theta)$.

1) By Schur complements, the inequalities (9) are transformed into the LMIs. For the convenience of design, $P$ is chosen as the form $P=\operatorname{diag}\left\{P_{1}, P_{2}\right\}$, where $P_{1}, P_{2}$ are some symmetric and positive definite matrices. The inequalities (9) are equivalent to the following matrix inequalities

$$
\left[\begin{array}{lll}
S_{11} & -P_{1} B_{i} K_{j} & 0 \\
-\left(B_{i} K_{j}\right)^{T} P_{1} & S_{22} & P_{2} \\
0 & P_{2} & -\rho^{2} I
\end{array}\right]<0, \quad i, j=1,2, \cdots, L
$$

Where $S_{11}=P_{1}\left(A_{i}+B_{i} K_{j}\right)+\left(A_{i}+B_{i} K_{j}\right)^{T} P_{1}+\sum_{l=1}^{r} \alpha_{l}^{-1} P_{1} A_{i l} A_{i l}^{T} P_{1}+\sum_{l=1}^{r} \alpha_{l} I+\frac{1}{\rho^{2}} P_{1} P_{1}+2 Q$,

$$
S_{22}=P_{2} A_{r}+A_{r}^{T} P_{2}+\sum_{l=1}^{r} \alpha_{l} I+2 Q .
$$

The matrix inequalities (12) imply $S_{11}<0$. Let $W=P_{1}^{-1}$ and $Y_{j}=K_{j} W . \quad S_{11}<0$ is equivalent to the LMIs with prescribed $Q$ and $\alpha_{l}(l=1, \cdots, r)$,

$$
\left[\begin{array}{ll}
S & W \\
W & -\left(\sum_{l=1}^{r} \alpha_{l} I+2 Q\right)^{-1}
\end{array}\right]<0, \quad i, j=1,2, \cdots, L
$$


where $S=A_{i} W+W A_{i}^{T}+B_{i} Y_{j}+\left(B_{i} Y_{j}\right)^{T}+\sum_{l=1}^{r} \alpha_{l}^{-1} A_{i l} A_{i l}^{T}+\left(\rho^{2}\right)^{-1} I$.

By solving the LMIs (13), $P_{1}$ and $K_{j}(j=1,2, \cdots, L)$ could be obtained. And then, by substituting $P_{1}$ and $K_{j}(j=1,2, \cdots, L)$ into (12), (12) becomes standard LMIs. We can easily solve $P_{2}$ from (12). Therefore, the common solution $P$ and $K_{j}(j=1,2, \cdots, L)$ could be found.

Remark 1: Either the matching condition or the upper bound is related to a large number of matrix operations. Without the matching condition and the upper bound, the dimension of the LMIs of this paper is reduced.

2) Fuzzy adaptive systems consist of four main components: fuzzy rule base, fuzzy inference engine, fuzzifier and defuzzifier [11]. The fuzzy rule base is composed of a collection of IF-THEN inference rules:

$R^{l}:$ IF $x_{1}$ is $A_{1}^{l}, \cdots, x_{n}$ is $A_{n}^{l}$ EŹTHEN $y$ is $G^{l}(l=1, \cdots p)$

where $A_{i}^{l}(i=1, \cdots, l)$ and $G^{l}(l=1, \cdots p)$ are fuzzy sets. The $k$ th element of $\Delta(x, x(t-\tau))$ is of the following form:

$$
\hat{\Delta}_{k}\left(x, x(t-\tau) \mid \theta_{k}\right)=\xi_{k}^{T}(x, x(t-\tau)) \theta_{k},
$$

where $\theta_{k}=\left(\theta_{k}^{1}, \cdots, \theta_{k}^{p}\right)^{T} \in R^{p}, \quad \xi_{k}^{T}(x, x(t-\tau))=\left(\xi_{k}^{1}, \cdots, \xi_{k}^{p}\right) \in R^{p}$,

$\xi_{k}^{l}=\prod_{i=1}^{n} \mu_{F_{i}^{l}}\left(x_{i}, x_{i}(t-\tau)\right) / \sum_{l=1}^{p} \prod_{i=1}^{n} \mu_{F_{i}^{l}}\left(x_{i}, x_{i}(t-\tau)\right), \mu_{F_{i}^{l}}\left(x_{i}, x_{i}(t-\tau)\right)=\mu_{F_{i}^{l}}\left(x_{i}\right) \prod_{j=1}^{r} \mu_{F_{i}^{l}}\left(x_{i}\left(t-\tau_{j}\right)\right)$,

and $\mu_{F_{i}^{l}}\left(x_{i}\right)(i=1,2, \cdots, n)$ are the membership functions.

In this paper, fuzzy logic systems are constructed to eliminate the uncertainties $\Delta(x, x(t-\tau))$. The approximation form is given as follows:

$$
\hat{\Delta}(x, x(t-\tau) \mid \Theta)=\Psi(x, x(t-\tau)) \Theta,
$$

where $\Psi(x, x(t-\tau))=\operatorname{diag}\left[\xi_{1}^{T}(x, x(t-\tau)), \cdots, \xi_{m}^{T}(x, x(t-\tau))\right], \Theta=\left[\theta_{1}^{T}, \theta_{2}^{T}, \cdots, \theta_{m}^{T}\right]^{T}$.

Define the optimal the parameter $\Theta^{*}$ as

$$
\Theta^{*}=\arg \min _{\Theta \in \Omega_{1}}\left[\sup _{x \in U_{1}}|\hat{u}(x, x(t-\tau) \mid \Theta)-\Delta(x, x(t-\tau))|\right],
$$

where $U_{1}=\left\{x \in R^{n}:\|x\| \leq N\right\}, \Omega_{1}=\left\{\Theta \in R^{p m}:\|\Theta\| \leq \mathrm{M}\right\} . U_{1}, \Omega_{1}$ denote the sets of suitable bounds on $x, \Theta$ respectively, $N, M$ are upper bounds.

The approximation error for the function $\Delta(x, x(t-\tau))$ can be expressed as

$$
\hat{\Delta}(x, x(t-\tau) \mid \Theta)-\Delta(x, x(t-\tau))=\Psi(x, x(t-\tau)) \tilde{\Theta}+w,
$$

where $\tilde{\Theta}=\Theta-\Theta^{*}$ the estimation error for $\Theta, w=\left[w_{1}, \cdots, w_{m}\right]^{T}$ is a residual term.

Remark 2: In order to guarantee $\|\Theta\| \leq \mathrm{M}$, the adaptive law (11) must be modified by the projection algorithm [11] as follows:

$$
\dot{\Theta}=\left\{\begin{array}{l}
\eta_{1} \Psi^{T}(x, x(t-\tau)) \bar{B}^{T} P \tilde{x}, \text { if }(\|\Theta\|<\mathrm{M}) \text { or }\left(\|\Theta\|=\mathrm{M} \text { and } \tilde{x}^{T} \mathrm{P} \bar{B} \Psi(x, x(t-\tau)) \Theta \leq 0\right) \\
\mathrm{P}_{\Theta}[.], \text { if }\|\Theta\|=\mathrm{M} \text { and } \tilde{x}^{T} \mathrm{P} \bar{B} \Psi(x, x(t-\tau)) \Theta>0
\end{array}\right.
$$

where $\mathrm{P}_{\Theta}[\cdot]=\eta_{1} \Psi^{T}(x, x(t-\tau)) \bar{B}^{T} P \tilde{x}-\eta_{1} \frac{\tilde{x}^{T} \mathrm{P} \bar{B} \Psi(x, x(t-\tau)) \Theta}{\|\Theta\|^{2}}$. 


\section{Stability analysis}

Substituting (11) into (11) yields

$$
\begin{gathered}
\dot{x}(t)=\sum_{i=1}^{L} \mu_{i}\left[A_{i} x(t)+\sum_{l=1}^{r} A_{i l} x\left(t-\tau_{l}\right)\right]+\sum_{i=1}^{L} \sum_{j=1}^{L} \mu_{i} \mu_{j} B_{i} K_{j}\left(x(t)-x_{r}(t)\right) \\
-B(\hat{u}(x, x(t-\tau) \mid \Theta)-\Delta(x, x(t-\tau)))+d .
\end{gathered}
$$

Let $\tilde{x}(t)=\left[x^{T}(t), x_{r}^{T}(t)\right]^{T}$, and $\bar{B}=\left[\begin{array}{ll}B^{T} & 0\end{array}\right]^{T}$. By using (11) and (17), a new extended closedloop system is as follows:

$$
\dot{\tilde{x}}(t)=\sum_{i=1}^{L} \sum_{j=1}^{L} \mu_{i} \mu_{j}\left[\bar{A}_{i j} \tilde{x}(t)+\sum_{l=1}^{r} \bar{A}_{i l} \tilde{x}\left(t-\tau_{l}\right)\right]+\bar{B}(-(\hat{u}(x, x(t-\tau) \mid \Theta)-\Delta(x, x(t-\tau)))+d \prime
$$

where $d \prime=\left[d^{T}, r^{T}(t)\right]^{T}$. When fuzzy logic systems $\hat{u}(x, x(t-\tau) \mid \Theta)$ could eliminate $\Delta(x, x(t-\tau))$, the closed-loop system (18) is stable.

By denoting $w^{\prime}=\left[\bar{w}^{T}, r^{T}(t)\right]^{T}, \bar{w}=\left[0, \cdots, d_{1}-w_{1}, \cdots, 0, \cdots, d_{m}-w_{m}\right]^{T}$ and using (14), the closed-loop system (18) could be rewritten as

$$
\dot{\tilde{x}}(t)=\sum_{i=1}^{L} \sum_{j=1}^{L} \mu_{i} \mu_{j}\left[\bar{A}_{i j} \tilde{x}(t)+\sum_{l=1}^{r} \bar{A}_{i l} \tilde{x}\left(t-\tau_{l}\right)\right]+\bar{B}(-\Psi(x, x(t-\tau)) \tilde{\Theta})+w \prime .
$$

From the above analysis, we have the following conclusion.

Theorem 1. Given a matrix $Q>0$, scalars $\rho>0, \alpha_{l}(l=1, \cdots, r)>0, \quad \eta_{1}>0$. If there exist matricesP $>0, K_{j}(j=1,2, \cdots, L)$ such that the inequalities (9) hold. If the updating law for fuzzy logic systems is chosen as (11). Then there exists a controller (11) with the fuzzy state feedback controller (8) and the adaptive compensator (10) such that, while maintaining all the closed-loop signals UUB, the following tracking performance(20) is achieved

$$
\int_{0}^{T}\left(x(t)-x_{r}(t)\right)^{T} Q\left(x(t)-x_{r}(t)\right) d t \leq \tilde{x}^{T}(0) P \tilde{x}(0)+\frac{1}{\eta_{1}} \tilde{\Theta}^{T}(0) \tilde{\Theta}(0)+\rho^{2} \int_{0}^{T}\left(w^{\prime} w^{T}\right) d t
$$

Proof: Consider the following Lyapunov-Krasoviskii candidate

$$
V=\frac{1}{2} \tilde{x}^{T} P \tilde{x}+\frac{1}{2} \sum_{l=1}^{r} \int_{t-\tau_{l}}^{t} \alpha_{l} \tilde{x}^{T}(v) \tilde{x}(v) d v+\frac{1}{2 \eta_{1}} \tilde{\Theta}^{T} \tilde{\Theta}
$$

where $\dot{V}=\dot{V}_{1}+\dot{V}_{2}, \dot{V}_{1}$ and $\dot{V}_{2}$ are given in (22) and (26), respectively.

$$
\begin{gathered}
\dot{V}_{1}=\frac{1}{2}\left(\sum_{i=1}^{L} \sum_{j=1}^{L} \mu_{i} \mu_{j}\left[\bar{A}_{i j} \tilde{x}(t)+\sum_{l=1}^{r} \bar{A}_{i l} \tilde{x}\left(t-\tau_{l}\right)\right]\right)^{T} P \tilde{x}(t)+\frac{1}{2} \tilde{x}^{T}(t) P\left(\sum _ { i = 1 } ^ { L } \sum _ { j = 1 } ^ { L } \mu _ { i } \mu _ { j } \left[\bar{A}_{i j} \tilde{x}(t)\right.\right. \\
\left.\left.+\sum_{l=1}^{r} \bar{A}_{i l} \tilde{x}\left(t-\tau_{l}\right)\right]\right)+\frac{1}{2} w^{\prime T} P x(t)+\frac{1}{2} x^{T}(t) P w \prime+\frac{1}{2} \sum_{l=1}^{r} \alpha_{l} \tilde{x}^{T}(t) \tilde{x}(t)-\frac{1}{2} \sum_{l=1}^{r} \alpha_{l} \tilde{x}^{T}\left(t-\tau_{l}\right) \tilde{x}\left(t-\tau_{l}\right) \\
\leq \frac{1}{2}\left(\sum_{i=1}^{L} \sum_{j=1}^{L} \mu_{i} \mu_{j}\left[\tilde{x}^{T}(t) \bar{A}_{i j}^{T} P \tilde{x}(t)+\tilde{x}^{T}(t) P \bar{A}_{i j} \tilde{x}(t)+\sum_{l=1}^{r} \alpha_{l}^{-1} \tilde{x}^{T}(t) P \bar{A}_{i l} \bar{A}_{i l}^{T} P \tilde{x}(t)+\sum_{l=1}^{r} \alpha_{l} \tilde{x}^{T}\left(t-\tau_{l}\right) \tilde{x}\left(t-\tau_{l}\right)\right]\right.
\end{gathered}
$$




$$
\begin{aligned}
& -\frac{1}{2}\left(\frac{1}{\rho} P x(t)-\rho w^{\prime}\right)^{T}\left(\frac{1}{\rho} P x(t)-\rho w^{\prime}\right)+\frac{1}{2} \rho^{2} w^{\prime} w^{\prime}+\frac{1}{2 \rho^{2}} \tilde{x}^{T}(t) P P \tilde{x}(t)+\frac{1}{2} \sum_{l=1}^{r} \alpha_{l} \tilde{x}^{T}(t) \tilde{x}(t) \\
& -\frac{1}{2} \sum_{l=1}^{r} \alpha_{l} \tilde{x}^{T}\left(t-\tau_{l}\right) \tilde{x}\left(t-\tau_{l}\right) \\
& \leq \frac{1}{2} \sum_{i=1}^{L} \sum_{j=1}^{L} \mu_{i} \mu_{j} \tilde{x}^{T}(t)\left(\bar{A}_{i j}^{T} P+P \bar{A}_{i j}+\sum_{l=1}^{r} \alpha_{l}^{-1} P \bar{A}_{i l} \bar{A}_{i l}^{T} P+\sum_{l=1}^{r} \alpha_{l} I+\frac{1}{\rho^{2}} P P\right) \tilde{x}(t)+\frac{1}{2} \rho^{2} w^{\prime} w^{\prime} .
\end{aligned}
$$

Substituting (9) into (22) yields

$$
\dot{V}_{1} \leq-\frac{1}{2} \tilde{x}^{T}(t) \bar{Q} \tilde{x}(t)+\frac{1}{2} \rho^{2} w^{T} w \prime
$$

By using (11),

$$
V_{2}=\left[\tilde{x}^{T} P \bar{B}\left(-(\Psi(x, x(t-\tau)) \tilde{\Theta})+\frac{1}{\eta_{1}} \tilde{\Theta}^{T} \dot{\Theta}\right]=0 .\right.
$$

From (23)-(24),

$$
\dot{V} \leq-\frac{1}{2} \tilde{x}^{T}(t) \bar{Q} \tilde{x}(t)+\frac{1}{2} \rho^{2} w^{T} w \prime
$$

When $\|\tilde{x}(t)\|>\frac{\rho}{\lambda_{\min }(\bar{Q})}\|w \prime\|, \dot{V}<0$.Thus, the closed-loop system consisting of $(1),(11),(8)$ and (10) is UUB .

Note that

$$
\int_{0}^{T}\left(x(t)-x_{r}(t)\right)^{T} Q\left(x(t)-x_{r}(t)\right) d t \leq \int_{0}^{T} \tilde{x}^{T}(t) \bar{Q} \tilde{x}(t) d t .
$$

Integrating the above equation (25) from $t=0$ to Tyields (20).

\section{Simulation example}

In this section, we provide an example to verify the effectiveness of the proposed control scheme.

Example: Consider the following 2-link manipulator system in [14]

$$
\ddot{q}(t)+C(q, \dot{q}) \dot{q}(t)+g(q)=B(q) u(t)+\sum_{i=1}^{r} \xi_{i}(t) q\left(t-\tau_{i}\right)+d \prime
$$

where $C(q, \dot{q})=H^{-1}(q) C \prime(q, \dot{q}), g(q)=H^{-1}(q) g^{\prime}(q), B(q)=H^{-1}(q), d \prime=H^{-1}(q) d, q=\left[q_{1}, q_{2}\right]^{T}$, $\xi_{i}(t)(i=1, \cdots, r)$ are uncertain and bounded, and $d$ is the external bounded disturbance.

The reference model is as follows:

$$
\dot{x}_{r}(t)=A_{r} x_{r}(t)+r(t)
$$

where $A_{r}=\operatorname{diag}\left\{A_{r 1}, A_{r 2}\right\}, A_{r 1}=A_{r 2}=\left[\begin{array}{ll}0 & 1 \\ -6 & -5\end{array}\right], r(t)=\left[0, r_{1}(t), 0, r_{2}(t)\right]^{T}, r_{1}(t)=r_{2}(t)=$ $3 \sin (2 t)$ 
Step1: Denote $x_{1}=q_{1}, x_{2}=\dot{q}_{1}, x_{3}=q_{2}$, and $x_{4}=\dot{q}_{2}$. Then, (26) can be written as a fourthdimension system. A nine-rule fuzzy T-S model is used to approximate the nonlinear 2-link manipulator system at $x_{1}=-\frac{\pi}{2}, 0, \frac{\pi}{2}$ and $x_{3}=-\frac{\pi}{2}, 0, \frac{\pi}{2}$, where

$$
\begin{aligned}
& A_{1}=\left[\begin{array}{llll}
0 & 1 & 0 & 0 \\
5.927 & -0.001 & -0.315 & -0.0000084 \\
0 & 0 & 0 & 1 \\
-6.859 & 0.002 & 3.155 & 0.0000062
\end{array}\right], A_{2}=\left[\begin{array}{llll}
0 & 1 & 0 & 0 \\
3.0428 & -0.0011 & -0.1791 & -0.0002 \\
0 & 0 & 0 & 1 \\
-3.5436 & 0.0313 & 2.5611 & 0.0000114
\end{array}\right], \\
& A_{3}=\left[\begin{array}{llll}
0 & 1 & 0 & 0 \\
6.2728 & 0.003 & 0.4339 & -0.0001 \\
0 & 0 & 0 & 1 \\
-9.1041 & 0.0158 & -1.0574 & -0.000032
\end{array}\right], A_{4}=\left[\begin{array}{llll}
0 & 1 & 0 & 0 \\
6.4535 & 0.0017 & 1.2427 & -0.0002 \\
0 & 0 & 0 & 1 \\
-3.1873 & 0.0306 & -5.1911 & -0.000018
\end{array}\right], \\
& A_{5}=\left[\begin{array}{llll}
0 & 1 & 0 & 0 \\
11.1336 & 0 & -1.8145 & 0 \\
0 & 0 & 0 & 1 \\
-9.0918 & 0 & 9.1638 & 0
\end{array}\right], A_{6}=\left[\begin{array}{llll}
0 & 1 & 0 & 0 \\
6.1702 & -0.001 & 1.687 & -0.0002 \\
0 & 0 & 0 & 1 \\
-2.3559 & 0.0314 & 4.5298 & -0.000011
\end{array}\right], \\
& A_{7}=\left[\begin{array}{llll}
0 & 1 & 0 & 0 \\
6.1206 & 0.0041 & 0.6205 & 0.0001 \\
0 & 0 & 0 & 1 \\
8.8794 & 0.0193 & -1.0119 & 0.000044
\end{array}\right], \quad A_{8}=\left[\begin{array}{llll}
0 & 1 & 0 & 0 \\
3.6421 & -0.0018 & 0.0721 & 0.0002 \\
0 & 0 & 0 & 1 \\
2.429 & -0.0305 & 2.9832 & -0.000019
\end{array}\right] \text {, } \\
& A_{9}=\left[\begin{array}{llll}
0 & 1 & 0 & 0 \\
6.2933 & -0.0009 & 0.2188 & -0.000012 \\
0 & 0 & 0 & 1 \\
-7.4649 & 0.0024 & 3.2693 & -0.0000092
\end{array}\right]
\end{aligned}
$$$$
A_{11}=A_{21}=A_{31}=A_{41}=A_{51}=A_{61}=A_{71}=A_{81}=A_{91}=\left[\begin{array}{llll}
0 & 0 & 0 & 0 \\
0.01 & 0 & 0 & 0 \\
0 & 0 & 0 & 0 \\
0 & 0 & 0 & 0
\end{array}\right] \text {, }
$$$$
A_{12}=A_{22}=A_{32}=A_{42}=A_{52}=A_{62}=A_{72}=A_{82}=A_{92}=\left[\begin{array}{llll}
0 & 0 & 0 & 0 \\
0 & 0 & 0 & 0 \\
0 & 0 & 0 & 0 \\
0 & 0 & 0.01 & 0
\end{array}\right] \text {, }
$$$$
B_{1}=\left[\begin{array}{llll}
0 & 1 & 0 & -1 \\
0 & -1 & 0 & 2
\end{array}\right]^{T}, B_{2}=\left[\begin{array}{llll}
0 & 0.5 & 0 & 0 \\
0 & 0 & 0 & 1
\end{array}\right]^{T}, B_{3}=\left[\begin{array}{llll}
0 & 1 & 0 & 1 \\
0 & 1 & 0 & 2
\end{array}\right]^{T},
$$$$
B_{4}=\left[\begin{array}{llll}
0 & 0.5 & 0 & 0 \\
0 & 0 & 0 & 1
\end{array}\right]^{T}, B_{5}=\left[\begin{array}{llll}
0 & 1 & 0 & -1 \\
0 & -1 & 0 & 2
\end{array}\right]^{T}, B_{6}=\left[\begin{array}{llll}
0 & 0.5 & 0 & 0 \\
0 & 0 & 0 & 1
\end{array}\right]^{T},
$$ 


$$
B_{7}=\left[\begin{array}{llll}
0 & 1 & 0 & 1 \\
0 & 1 & 0 & 2
\end{array}\right]^{T}, B_{8}=\left[\begin{array}{llll}
0 & 0.5 & 0 & 0 \\
0 & 0 & 0 & 1
\end{array}\right]^{T}, B_{9}=\left[\begin{array}{llll}
0 & 1 & 0 & -1 \\
0 & -1 & 0 & 2
\end{array}\right]^{T} .
$$

The membership functions are adopted as the triangle type.

Step 2: On the basis of Theorem1, with $\alpha_{1}=0.005, \alpha_{2}=0.005$, and $\rho=1$,we have

$$
\begin{aligned}
K_{1} & =\left[\begin{array}{llll}
-76.9685 & -42.9566 & -19.6919 & -8.9116 \\
6.0025 & -0.4619 & -51.4252 & -25.0336
\end{array}\right], \\
K_{2} & =\left[\begin{array}{llll}
-77.7828 & -42.8754 & -13.6211 & -5.9413 \\
8.7179 & 1.2251 & -50.6614 & -24.6859
\end{array}\right], \\
K_{3} & =\left[\begin{array}{llll}
-76.8347 & -42.9089 & -19.8204 & -8.9785 \\
5.8595 & -0.5257 & -51.3739 & -25.0109
\end{array}\right], \\
K_{4} & =\left[\begin{array}{llll}
-77.7828 & -42.8754 & -13.6211 & -5.9413 \\
8.7179 & 1.2251 & -50.6614 & -24.6859
\end{array}\right], \\
K_{5} & =\left[\begin{array}{llll}
-77.7828 & -42.8754 & -13.6211 & -5.9413 \\
8.7179 & 1.2251 & -50.6614 & -24.6859
\end{array}\right], \\
K_{6} & =\left[\begin{array}{llll}
-77.7828 & -42.8754 & -13.6211 & -5.9413 \\
8.7179 & 1.2251 & -50.6614 & -24.6859
\end{array}\right], \\
K_{7} & =\left[\begin{array}{llll}
-79.8424 & -43.4072 & -6.0780 & -2.2626 \\
12.7745 & 3.6898 & -50.2150 & -24.4989
\end{array}\right], \\
K_{8} & =\left[\begin{array}{llll}
-77.7828 & -42.8754 & -13.6211 & -5.9413 \\
8.7179 & 1.2251 & -50.6614 & -24.6859
\end{array}\right], \\
K_{9}= & {\left[\begin{array}{llll}
-80.1162 & -43.5088 & -5.8152 & -2.1328 \\
13.0509 & 3.8147 & -50.3242 & -24.5472
\end{array}\right], }
\end{aligned}
$$

Step 3: In fuzzy adaptive compensator, the membership functions are selected as

$$
\begin{gathered}
\mu_{F_{i}^{1}}\left(x_{i}\right)=\frac{1}{1+\exp \left[5\left(x_{i}+0.8\right)\right]}, \mu_{F_{i}^{2}}\left(x_{i}\right)=\exp \left[-\left(x_{i}+0.6\right)^{2}\right], \mu_{F_{i}^{3}}\left(x_{i}\right)=\exp \left[-\left(x_{i}+0.4\right)^{2}\right], \\
\mu_{F_{i}^{4}}\left(x_{i}\right)=\exp \left[-\left(x_{i}\right)^{2}\right], \mu_{F_{i}^{5}}\left(x_{i}\right)=\exp \left[-\left(x_{i}-0.4\right)^{2}\right], \mu_{F_{i}^{6}}\left(x_{i}\right)=\exp \left[-\left(x_{i}-0.6\right)^{2}\right], \\
\mu_{F_{i}^{7}}\left(x_{i}\right)=\frac{1}{1+\exp \left[5\left(x_{i}-0.8\right)\right]}, \quad i=1,2, \cdots, 4 .
\end{gathered}
$$

Step 4: Some parameters are choose as

$$
\begin{gathered}
\eta_{1}=10, r=2, \quad \tau_{1}=0.5, \tau_{2}=1, \xi_{1}(t)=5+20 \sin (5 \mathrm{t}), \operatorname{and} \xi_{1}(t)=1+15 \cos (5 \mathrm{t}), \\
\Theta(0)=[0.2,0.2,0.2,0.2,0.2,0.2,0.2,0.2,0.2,0.2,0.2,0.2,0.2,0.2,0.2], \\
\left(x_{1}(0), x_{2}(0), x_{3}(0), x_{4}(0), x_{r 1}(0), x_{r 2}(0), x_{r 3}(0), x_{r 4}(0)\right)=(0.4,0,-0.4,0,0,0,0,0) .
\end{gathered}
$$

By using the method in Theorem 1, the tracking performances of $x_{1}(t), x_{2}(t), x_{3}(t), x_{4}(t)$ are shown in Fig.1,and the control efforts $u_{1}(t)$ and $u_{2}(t)$ are given in Fig.2,respectively. 


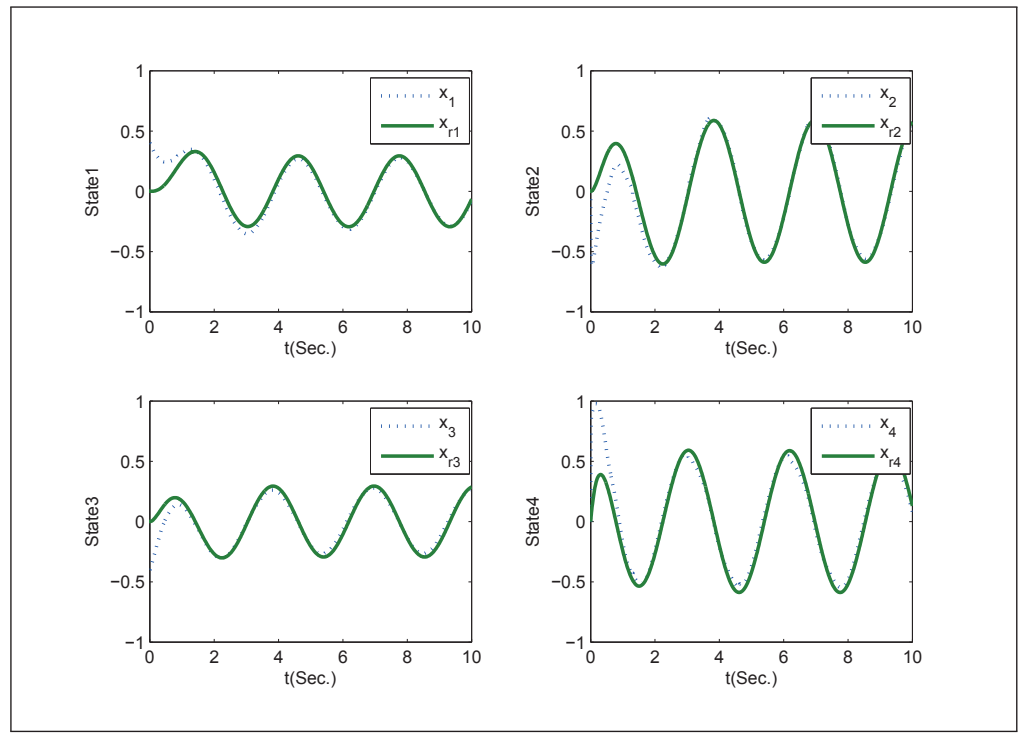

Figure 1: The responses of $x_{1}, x_{2}, x_{3}, x_{4}, x_{r 1}, x_{r 2}, x_{r 3}$ and $x_{r 4}$

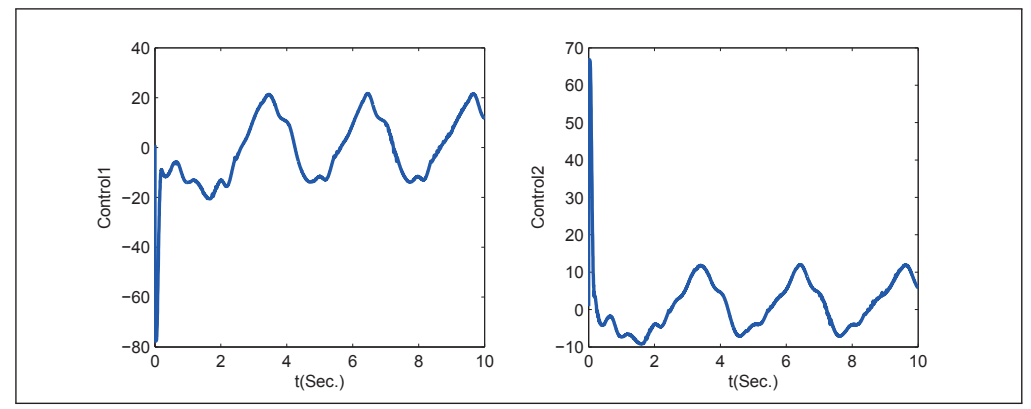

Figure 2: The control inputs $u_{1}, u_{2}$

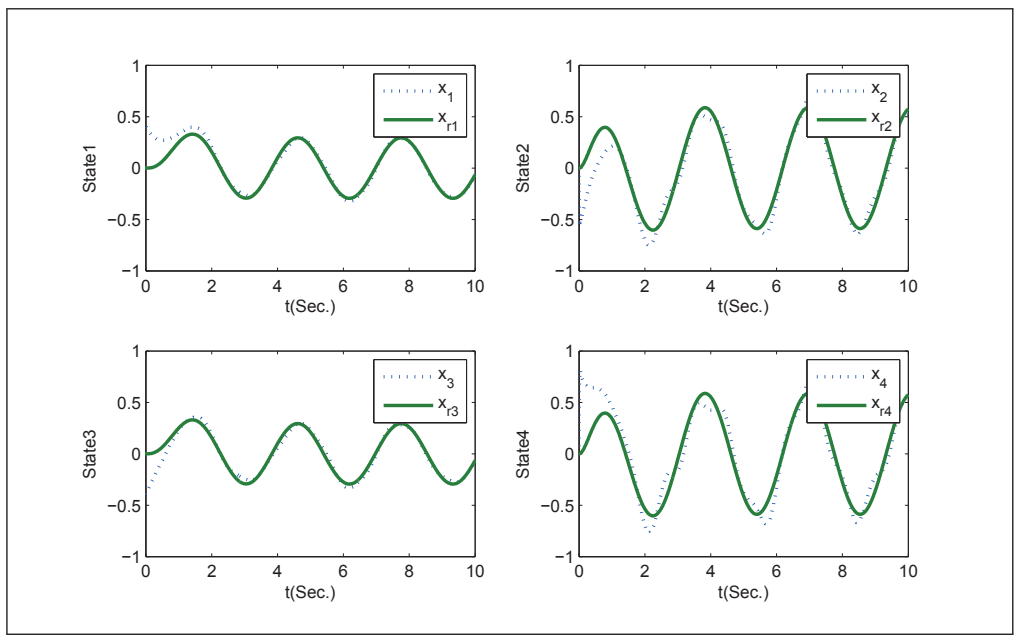

Figure 3: The responses of $x_{1}, x_{2}, x_{3}, x_{4}, x_{r 1}, x_{r 2}, x_{r 3}$ and $x_{r 4}$ 

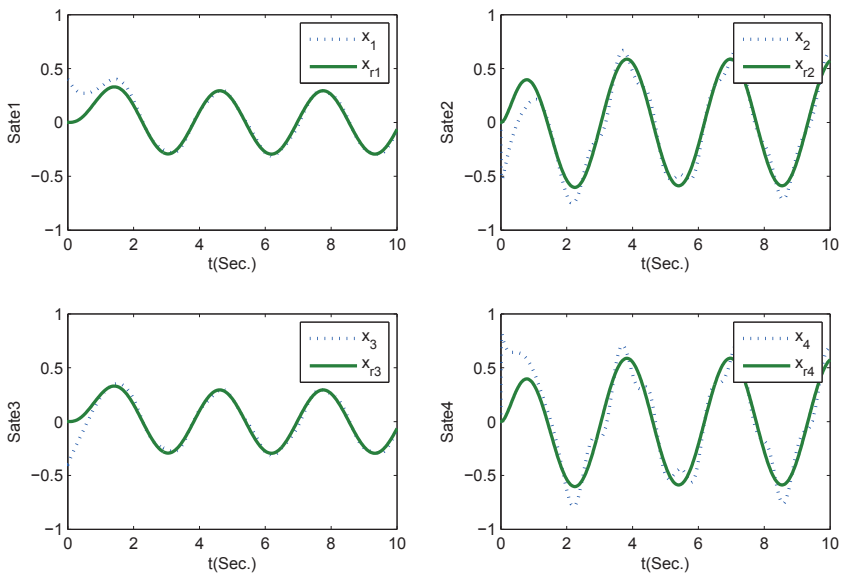

Figure 4: The responses of $x_{1}, x_{2}, x_{3}, x_{4}, x_{r 1}, x_{r 2}, x_{r 3}$ and $x_{r 4}$
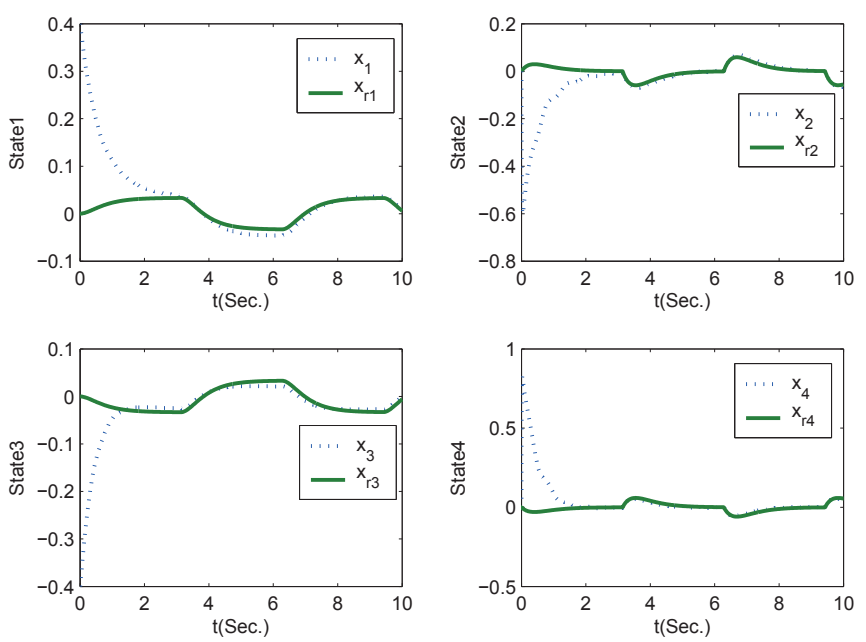

Figure 5: The responses of $x_{1}, x_{2}, x_{3}, x_{4}, x_{r 1}, x_{r 2}, x_{r 3}$ and $x_{r 4}$

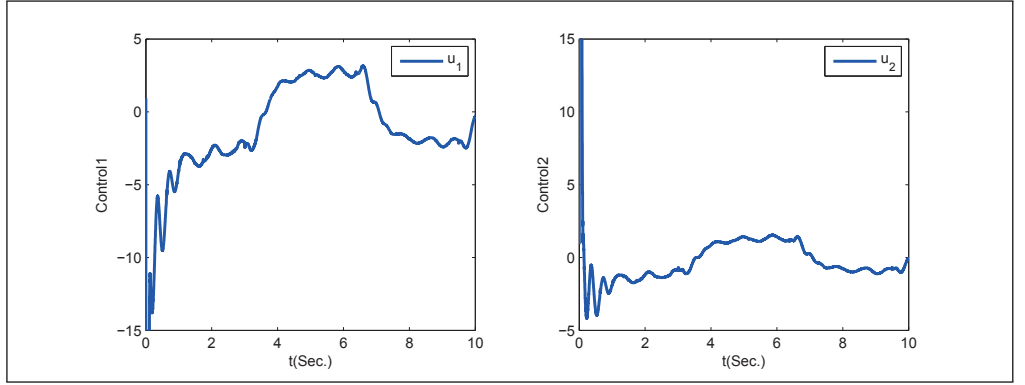

Figure 6: The control inputs $u_{1}, u_{2}$ 
When $\tau_{1}=1, \tau_{2}=1$, simulation results are shown in Fig.3.When $\tau_{1}=1, \tau_{2}=2$, simulation results are shown in Fig.4.

When $r_{1}(t)$ and $r_{2}(t)$ are square waves having an amplitude \pm 0.2 with a period of $2 \pi$, the tracking performances of $x_{1}(t), x_{2}(t), x_{3}(t), x_{4}(t)$ are shown in Fig. 5 , and the control efforts $u_{1}(t)$ and $u_{2}(t)$ are given in Fig.6.

Simulation results illustrate that the proposed controller design is effective and feasible.

\section{Conclusion}

Based on fuzzy technique, a novel tracking control scheme is presented for uncertain nonlinear time-delay system. As main contribution of this paper, we design a novel fuzzy tracking controller, which is independent of the matching condition or the upper bound for the uncertainties. Furthermore, the tracking control design for discrete nonlinear systems is also developed.

\section{Acknowledgment}

This work was supported by the National Natural Science Foundation of China(61203320,61273128), Shandong Natural Science Foundation(ZR2014FL023) and the project of Shandong Province higher educational science and technology program(J14LN70).

\section{Bibliography}

[1] T. Takagi; M. Sugeno. (1985); Fuzzy identification of systems and its applications to modeling and control, IEEE Transactions on Systems, Man, and Cybernetics, ISSN 0018-9472, SMC-15(1):116-132.

[2] J.-W. Wang; H.-N. Wu; H.-X. Li. (2011); Distributed fuzzy control design of nonlinear hyperbolic PDE systems with application to nonisothermal plug-flow reactor, IEEE Transactions on Fuzzy Systems, ISSN 1063-6706, 19(3): 514 - 526.

[3] D.W. Kim;H.J. Lee. (2012); Sampled-data observer-based output-feedback fuzzy stabilization of nonlinear systems: Exact discrete-time design approach, Fuzzy Sets and Systems, ISSN 0165-0114, 201(16): 20-39.

[4] G.K. Koo;J.B. Park;Y.H. Joo. (2013); Guaranteed cost sampled-data fuzzy control for nonlinear systems: a continuous-time Lyapunov approach, IET Control Theory and Applications, ISSN 1751-8644, 7(13): 1745-1752.

[5] Y.-S. Zhang;S.-Y. Xu;Y. Zou;J.-J. Lu. (2011); Delay-dependent robust stabilization for uncertain discrete-time fuzzy Markovian jump systems with mode-dependent time delays, Fuzzy Sets and Systems, ISSN 0165-0114, 164(1):66-81.

[6] J. Yoneyama. (2012); Robust sampled-data stabilization of uncertain fuzzy systems via input delay approach, Information Sciences, ISSN 0020-0255, 198 (1): 169-176.

[7] J. Yoneyama. (2013); Robust H8 filtering for sampled-data fuzzy systems, Fuzzy Sets and Systems, ISSN 0165-0114, 217(16) : 110-129. 
[8] Z.-Y. Xi;G. Feng;T. Hesketh. (2011); Piecewise Integral Sliding-Mode Control for T-S Fuzzy Systems, IEEE Transactions on Fuzzy Systems, ISSN 1063-6706, 19(1): 65-74.

[9] J. Chen;F.Sun;Y.Yin;C.Hu. (2011); State feedback robust stabilization for discrete-time fuzzy singularly perturbed systems with parameter uncertainty, IET Control Theory and Applications, ISSN 1751-8644, 5(10): 1195 - 1202.

[10] C.-H. Lien;J.-D. Chen;K.-W. Yu;L.-Y.Chung. (2012); Robust delay-dependent H8 control for uncertain switched time-delay systems via sampled-data state feedback input, Computers and Mathematics with Applications, ISSN 0898-1221, 64(5):1187-1196.

[11] L.-X. Wang. (1993); Stable adaptive fuzzy control of nonlinear systems, IEEE Transactions on Fuzzy Systems, ISSN 1063-6706,1(3):146-155.

[12] W.-S. Chen;Z.-Q. Zhang. (2010); Globally stable adaptive backstepping fuzzy control for output-feedback systems with unknown high-frequency gain sign, Fuzzy Sets and Systems, ISSN 0165-0114,161(6): 821-836.

[13] Z.-B. Du;T.-C. Lin;V. E. Balas. (2012); A new approach to nonlinear tracking control based on fuzzy approximation, International Journal of Computers, Communications and Control, ISSN 1841-9836,7(1):61-72.

[14] W.-S. Yu. (2004); Tracking-based adaptive fuzzy-neural control for MIMO uncertain robotic systems with time delays, Fuzzy Sets and Systems, ISSN 0165-0114,146(3): 375-401. 